\title{
Atualização do Perfil Epidemiológico da Hantavirose no Brasil
}

\author{
Luciene da Silva Guedes ${ }^{1}$, Bruno Silva Milagres², Stefan Vilges de Oliveira ${ }^{3}$
}

\begin{abstract}
RESUMO
O objetivo deste é realizar uma atualização do perfil epidemiológico da hantavirose no Brasil. Para isso, foi realizado um estudo descritivo das notificações de casos de hantavirose ao Sistema de Informação de Agravos de Notificação (Sinan) do Ministério da Saúde no período de 2007 a 2017. Foram realizadas 16.260 notificações de casos de hantavirose ao Sinan, com $8 \%$ confirmados, e as regiões mais afetadas são a Sul, a Sudeste e a Centro-Oeste. A faixa etária mais atingida foi de 20 a 49 anos, e o sexo masculino. Os sintomas da doença são inespecíficos, quando o mais frequente foi a febre, e o diagnóstico confirmatório de casos ocorreu por exames laboratoriais. A maioria dos casos relatou que a possível infecção aconteceu em atividades laborais, na área rural e nos locais próximos ao habitat do hospedeiro. Quase todos os casos foram hospitalizados e os óbitos são em torno de $40 \%$. Os dados obtidos reforçam a necessidade de intensificar a vigilância da doença no país, visando a reduzir o adoecimento e a morte por hantavirose.
\end{abstract}

Palavras-chave: Hantavírus. Síndrome cardiopulmonar por hantavírus. Vigilância epidemiológica.

\section{UPDATE ON THE EPIDEMIOLOGICAL PROFILE OF HANTAVIROSIS IN BRAZIL}

\begin{abstract}
The objective of this study is to update the epidemiological profile of hantavirosis in Brazil. For this, a descriptive and retrospective study of the reports of cases of hantavirosis was carried out to the Information System of Notification Diseases (Sinan) of the Ministry of Health from 2007 to 2017. There were 16,260 reports of hantavirosis cases at Sinan, where 8\% were confirmed and the most affected regions are South, Southeast and Center West. The most affected age group was 20 to 49 years and males. The symptoms of the disease are nonspecific, where the most frequent was fever and the confirmatory diagnosis of cases occurred by laboratory tests. Most of the cases reported that the possible infection occurred in work activities, in the rural area and in the places close to the host's habitat. Almost all cases were hospitalized, and deaths were around $40 \%$. The data obtained reinforce the need to intensify surveillance of the disease in the country, with a view to reducing sickness and death due to hantavirosis.
\end{abstract}

Keywords: Hantavirus. Hantavirus cardiopulmonary syndrome. Epidemiologic surveillance.

Recebido em: 26/5/2018

Alterações requeridas em: 29/5/2019

Aceito em: $3 / 6 / 2019$

\footnotetext{
Graduação em Biomedicina pelo Centro Universitário de Brasília. Atuou como técnica em Enfermagem do Hospital das Forças Armadas (2005-2010), Cabo Enfermeira na Marinha do Brasil (2009-2010) e técnica em Enfermagem na Secretaria de Estado de Saúde do Distrito Federal na área de Vigilância Epidemiológica. luguedes1@gmail.com

Graduação (Bacharelado e Licenciatura) em Ciências Biológicas pela Universidade Federal de Ouro Preto (2002 e 2003$)$. Mestrado em Medicina Preventiva pela Medicina Veterinária da Universidade Federal de Viçosa (2004) e Doutorado em Biologia Celular e Molecular pelo Núcleo de Pesquisa em Ciências Biológicas da Universidade Federal de Ouro Preto (2010). Treinamento em nível de Pós-Doutorado (Laboratório de Parasitologia e Epidemiologia Molecular do Departamento de Bioquímica e Biologia Molecular - Universidade Federal de Viçosa) e em epidemiologia pelo Episus (Programa de Treinamento em Epidemiologia Aplicada aos Serviços do SUS) - Ministério da Saúde. Experiência na área de Biologia Geral, com ênfase em Epidemiologia Molecular e Biologia Molecular, trabalhando com febre maculosa.bruno.milagres@saude.gov.br

${ }^{3}$ Graduação em Ciências Biológicas (Licenciatura) pela Universidade da Região da Campanha (2005). Especialização em Ecologia pela Universidade Federal do Rio Grande (2009). Mestrado (2013) e Doutorado (2017) em Medicina Tropical, nas áreas de Epidemiologia e Biologia das Doenças Infecciosas e Parasitárias pela Universidade de Brasília. Consultor técnico da Organização Pan Americana da Saúde (Opas/OMS). - Epidemiologista para a Secretaria de Vigilância em Saúde (SVS) do Ministério da Saúde (MS) do Brasil (2010-2018). Professor-adjunto A do Departamento de Saúde Coletiva da Faculdade de Medicina da Universidade Federal de Uberlândia. stefan@ufu.br
} 


\section{INTRODUÇÃO}

A hantavirose é uma doença zoonótica, aguda, causada por vírus RNA, da família Hantaviridae pertencente ao gênero Orthohantavirus (ICTV, 2017). A infecção humana pode variar desde assintomática a uma doença aguda inespecífica febril e autolimitada (MATTAR et al., 2015).

No Brasil, a forma clínica observada é denominada de Síndrome Cardiopulmonar por Hantavírus (SCPH) (MS, 2013). A SCPH caracteriza-se pelo grave comprometimento cardiovascular e respiratório que se desenvolve em duas fases: a) a fase prodrômica ou inespecífica, com duração de três a quatro dias, caracterizada por hipertermia, mialgia, cefaleia, dor abdominal, náuseas, vômitos, diarreia, astenia e petéquias, sintomas estes pouco específicos e que dificultam seu diagnóstico no período inicial, promovendo que seja confundida facilmente com outras doenças virais (FERREIRA, 2003; MATTAR et al., 2015); b) fase cardiopulmonar, que é iniciada com a tosse seca, acompanhada por taquicardia, taquidispneia e hipoxemia, quando a dispneia, em geral, surge no 3 은 ou 4 - dias por causa da progressiva infiltração de líquidos e proteínas no interstício e nos alvéolos pulmonares, resultando, dessa maneira, na hipovolemia e levando à hipotensão arterial e depressão da função miocárdica (FERREIRA, 2003; MATTAR et al., 2015).

A SCPH não tem tratamento específico e as condutas de assistência médica estão pautadas em medidas de suporte em unidades de terapia intensiva. Desta forma, a suspeição clínica e epidemiológica e o diagnóstico oportuno são fundamentais para reduzir as chances de morte pela doença (WILLEMANN; OLIVEIRA, 2014; MATTAR et al., 2015).

Os hantavírus possuem como reservatórios naturais alguns roedores silvestres, que podem eliminar o vírus pela urina, saliva e fezes (DE OLIVEIRA et al., 2013). Morcegos, insetívoros e marsupiais também podem se infectar, mas até o momento a participação destes no ciclo epidemiológico da doença ainda não foi bem-estabelecida (DE OLIVEIRA, R. C. et al., 2014).

$A$ inalação de aerossóis formados pelas excretas e secreções de roedores silvestres infectados é a principal forma de transmissão do vírus, podendo ocorrer também pela mordedura e arranhadura, ingestão de alimentos infectados por saliva ou urina destes animais, e, menos frequentemente, por meio de mãos contaminadas levadas à boca. A transmissão é possibilitada por procedimentos que permitam a aerossolização das partículas virais presentes no ambiente infestado por roedores (FERREIRA, 2003).

Sabe-se que diferentes espécies de hantavírus já foram identificadas em roedores de várias espécies (DE OLIVEIRA, R. C. et al., 2014). No Brasil, as variantes virais mais prevalentes são: Juquitiba, que ocorre em roedores silvestres da Floresta Atlântica; Araraquara, comum aos roedores do cerrado e caatinga; Castelo dos Sonhos, oriundos dos roedores do cerrado e Floresta Amazônica; e Anajatuba, encontrado em roedores da Floresta Amazônica e regiões alagadas do Maranhão (FIRTH et al., 2012).

A SCPH é considerada uma doença emergente, pois foi reconhecida nos Estados Unidos somente em 1993 (NICHOL et al., 1993; KHAN et al., 1996), acometendo rapidamente outros países da América Central e do Sul. Também, no mesmo ano, no Estado de São Paulo, foram reconhecidos os primeiros casos do Brasil (SILVA et al., 1997).

De acordo com De Oliveira, S. V. et al. (2014), a relevância em saúde pública da hantavirose está relacionada ao alto coeficiente de letalidade da doença, sobretudo nas regiões Sul, Sudeste e Centro-Oeste. Desde a sua emergência no país, a SCPH vem ampliando a sua área de ocorrência, incluindo novas regiões como áreas endêmicas (OLIVEIRA et al., 2015; VARGAS et al., 2016). Por esse motivo, é de notificação compulsória e imediata aos serviços de saúde, devendo todo caso ser registrado e investigado no âmbito do Sistema de Único de Saúde (SUS) (MS, 2013).

O diagnóstico da doença é complexo e requer a avaliação clínica, epidemiológica e laboratorial (MS, 2013; MATTAR et al., 2015). Assim sendo, o SUS necessita sistematicamente da disponibilização de informações epidemiológicas atualizadas, visando a ampliar o entendimento e contribuir para as ações de vigilância da referida doença (MS, 2013).

A última informação epidemiológica sobre a SCPH foi publicada por De Oliveira, S. V. et al., (2014), que analisaram o perfil epidemiológico da hantavirose no Brasil no período de 2007 a 2012. Nos anos recentes, no entanto, registros da doença em novas áreas do país destacam a necessidade de realizar uma nova avaliação e atualização epidemiológica do perfil da hantavirose, incluindo registros das novas regiões de ocorrência do país (VARGAS et al., 2016).

Dessa maneira, o presente estudo tem por objetivo realizar uma atualização do perfil epidemiológico dos casos de hantavirose no Brasil no período de 2007 a 2017.

\section{MATERIAIS E MÉTODOS}

Trata-se de um estudo epidemiológico descritivo do período de 2007 a 2017. O presente trabalho analisa o perfil dos casos de hantavirose em todas as Unidades Federadas (UF) do Brasil. Como fonte de da- 
Figura 1 - Casos de hantavirose no Brasil notificados ao Sinan no período de 2007 a 2017?
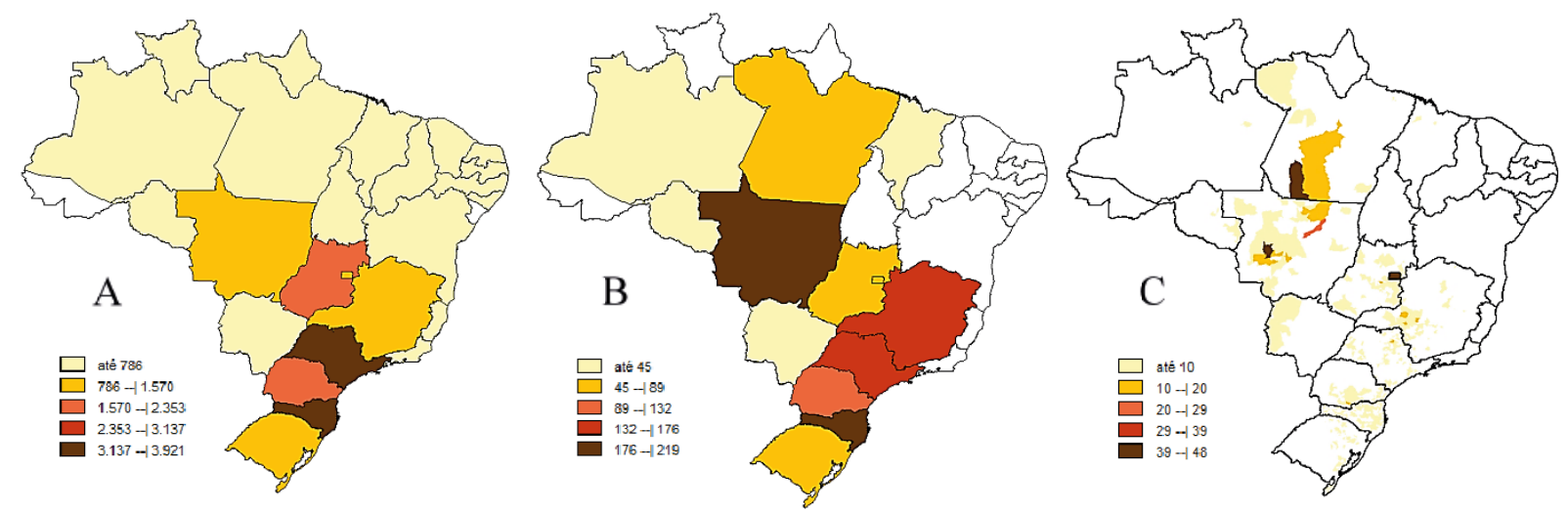

A - Distribuição dos casos notificados de hantavirose segundo a Unidade Federada (UF) da notificação. B - Distribuição dos casos confirmados de hantavirose segundo a UF da infecção. C - Distribuição dos casos confirmados de hantavirose segundo o município provável da infecção.

dos para este estudo, foi utilizada a base de dados do Sistema de Informação de Agravos de Notificação (Sinan-Net), do Ministério da Saúde do Brasil. $O$ acesso à base de dados foi em 22 de maio de 2018.

Para análise utilizou-se as variáveis demográficas [município; unidade federada do registro; sexo; faixa etária estratificada em anos: <1 Ano; 1-4; 5-9; 10-14; 15-19; 20-34; 35-49; 50-64; 65-79; 80 e +], epidemiológicas [data de início de sintomas; antecedentes epidemiológicos; critério de diagnóstico], clínicas e de hospitalização [sinais e sintomas e evolução do caso; hospitalização - sim ou não] (MS, 2013).

Foi considerado como caso confirmado de hantavirose o caso suspeito, segundo os critérios do Ministério da Saúde do Brasil, e com o seguinte resultado de exame laboratorial: I) sorologia reagente para anticorpos séricos específicos para hantavírus da classe IgM, ou II) imunohistoquímica de tecidos positiva (identificação de antígenos específicos contra hantavírus), ou III) RT-PCR positivo para hantavirose (MS, 2013).

Foram feitas análises de estatística descritiva e os resultados apresentados por meio de números brutos, com medidas de proporção e de tendência central. As análises epidemiológicas foram realizadas pelos softwares Microsoft Excel 2013 e Tabwin 32.

0 estudo foi realizado com dados secundários, apresentados de forma coletiva, e não foram acessadas informações nominais ou que pudessem identificar algum indivíduo; desta forma não necessitou de apreciação do Comitê de Ética em Pesquisa.

\section{RESULTADOS E DISCUSSÃO}

Foram notificados ao Sinan, no período de 2007 a 2017, 16.260 casos de hantavirose (Figura 1-A). Destes, foram confirmados $8 \%(1.242 / 16.260)$, distribuí- dos em 13 UFs (Figura 1-B) e em 7,71\% (430/5570) dos municípios brasileiros (Figura 1-C). As UFs com maior concentração de casos foram a Região Sul $(29,62 \%$ - 368/1.242) e a Região Centro Oeste $(28,42 \%$ $353 / 1.242$ ). O número médio de casos confirmados por ano foi de 113, e o coeficiente médio de letalidade foi de 41\%; maior nos anos de 2011 (51\%) e no ano de 2016 (53\%), conforme descrito na Figura 5. O total de 495 casos com o diagnóstico de hantavirose evoluíram para o óbito.

A faixa etária mais acometida foi de 20 a 49 anos (72\% - 897/1.242), e o intervalo de idade variou de zero meses a mais de 80 anos, e deste total, $76 \%$ (939/1.242) foram do sexo masculino, como pode-se observar na Figura 2.

Figura 2 - Casos confirmados de hantavirose segundo a faixa etária estratificada e o sexo - Brasil, 2007 a 2017

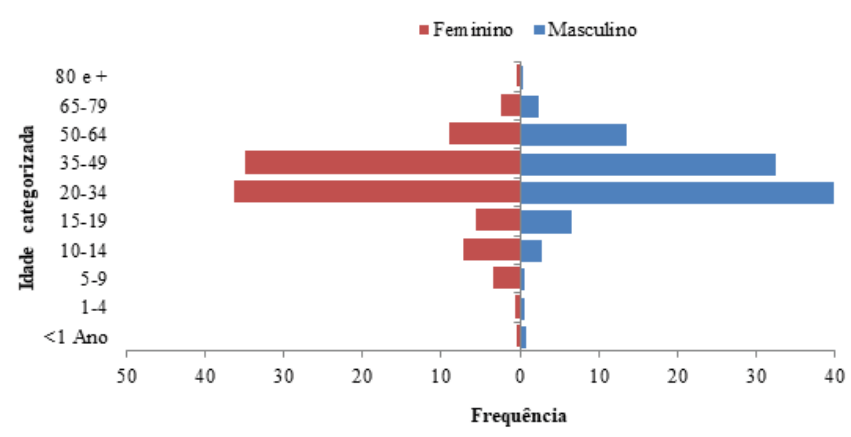

Os dados observados corroboram a literatura existente (NUNES et al., 2011; DE OLIVEIRA, S. V. et al., 2014; OLIVEIRA et al., 2015), quando se sugere que a hantavirose é um agravo relacionado à atividade laboral, pois acomete indivíduos em maior número do sexo masculino, nas idades apontadas como as mais produtivas (NUNES et al., 2011; DE OLIVEIRA, S. V. et al., 2014; OLIVEIRA et al., 2015). 
É necessário ressaltar que os indivíduos do sexo masculino, por serem predominantes nas atividades rurais, foram tanto mais acometidos pela doença quanto foram a óbito em maior frequência $(74 \%$ $368 / 495)$, embora o sexo feminino tenha apresentado maior coeficiente de letalidade $(42 \%-127 / 303)$ quando comparado aos homens (39\% - 368/939). A maior letalidade em mulheres pode ser explicada pela menor frequência de registros de casos, bem como menor suspeita clínica e epidemiológica.

Na fase prodrômica $(30 \%-368 / 1.242)$, os sintomas primários e mais prevalentes foram febre, dispneia, cefaleia, mialgia e vômitos/náusea. Os sintomas mais severos ocorrem na fase da SCPH $(68 \%$ - 850/1.242), com início aproximadamente até no 70 dia após essa fase, e consistem em tosse seca, taquicardia, taquidispneia, edema pulmonar, hipotensão e colapso circulatório (MS, 2013; MATTAR et al., 2015). A descrição da frequência dos principais sinais e sintomas dos casos confirmados de hantavirose é apresentada na Figura 3.

Figura 3 - Sinais e sintomas dos casos confirmados de hantavirose - Brasil, 2007 a 2017

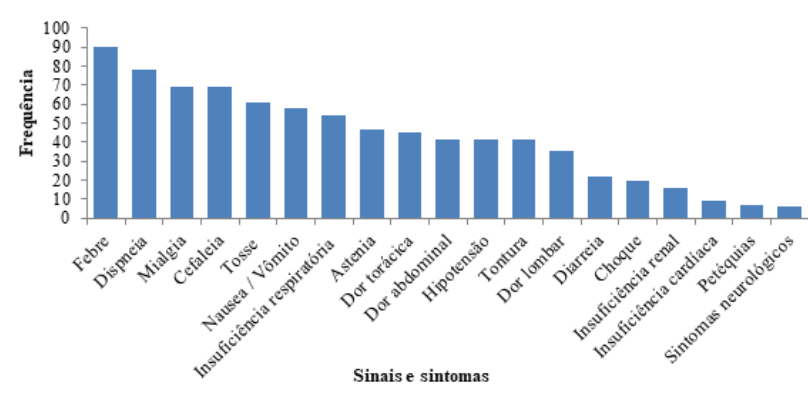

Esses sintomas inespecíficos descritos podem ser confundidos com muitas outras doenças endêmicas prevalentes, o que dificulta o diagnóstico diferencial e o tratamento de suporte precoce (MS, 2013; MATTAR et al., 2015). Além disso, o tempo entre o início dos sintomas e a piora do quadro é significantemente curto, sendo agravado pela demora dos pacientes em buscar auxílio médico (MS, 2013; MATTAR et al., 2015). Essa situação pode ser observada quando se verifica que cerca de 90\% (1.129/1.242) dos pacientes necessitaram de assistência hospitalar.

No presente estudo, os casos de hantavirose foram confirmados predominantemente pelo critério laboratorial $(92,59 \%-1.150 / 1.242)$, seguidos pelo critério clínico epidemiológico $(5,48 \%$ - 68/1.242), estando de acordo com os critérios preconizados pelo Ministério da Saúde (MS, 2013).
Em relação aos antecedentes epidemiológicos, a maioria dos casos ocorreu em indivíduos que exerciam atividades profissionais relacionadas à limpeza de galpões $(46 \%$ - 578/1.242), desmatamento $(40 \%$ $502 / 1.242$ ) e com algum tipo de contato direto com o rato $(39 \%-473 / 1.242)$ (Figura 4$)$, corroborando os dados epidemiológicos que antecederam este estudo (NUNES et al., 2011; DE OLIVEIRA, S. V. et al., 2014; OLIVEIRA et al., 2015).

Figura 4 - Antecedentes epidemiológicos dos casos confirmados de hantavirose

(60 dias antes dos sintomas) - Brasil, 2007 a 2017

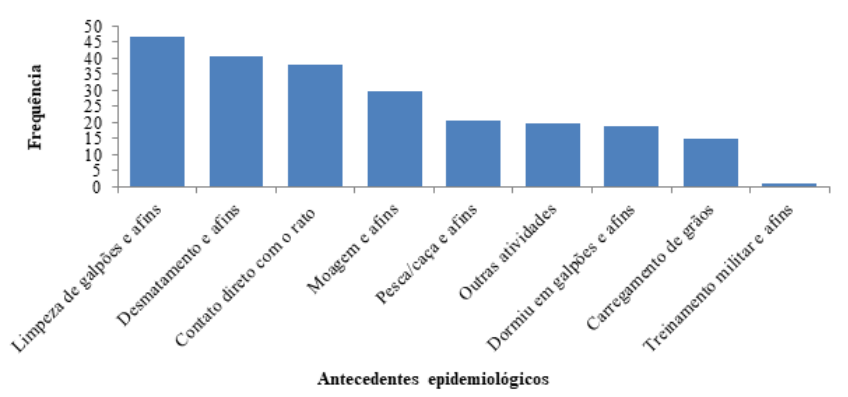

Os resultados demonstram que a partir de 2016 o número de casos confirmados reduziu (Figura 5 ), fato este que precisa ser mais bem explorado em análises regionalizadas para ampliar o entendimento deste perfil, identificando áreas prioritárias para intervenções. Embora, no entanto, o número de casos seja baixo quando comparado a outras doenças infecciosas endêmicas e mais incidentes, como dengue, doença de chagas e leishmaniose, o impacto da hantavirose é expresso no coeficiente elevado de letalidade (DE OLIVEIRA, S. V. et al., 2014).

É possível que tanto os dados de letalidade quanto o número de registros possam estar subestimados em razão da subnotificação de informações aos sistemas de vigilância epidemiológica (SANTOS; GARRETT, 2005).

Figura 5 - Número de casos confirmados de hantavirose e o coeficiente de letalidade da doença no Brasil - 2007 a 2017

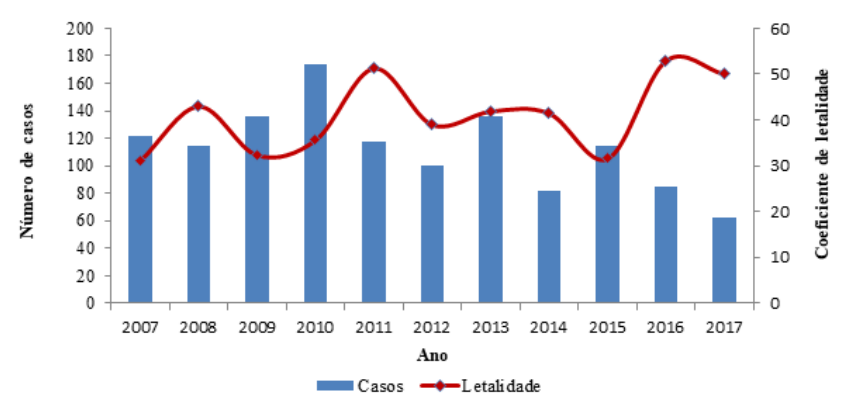


As regiões com maior frequência de notificações são as áreas onde a doença ocorre há mais tempo (Regiões Sul, Sudeste e Centro-Oeste) (Figura 1-A). Desta forma, torna-se necessário o fortalecimento dos serviços de vigilância epidemiológica e de assistência médica, buscando oportunizar o reconhecimento da circulação da doença em áreas com baixa frequência de notificações, priorizando áreas consideradas silenciosas da Região Nordeste do país (Figuras $1 \mathrm{~B}$, C). No período avaliado por este estudo, observa-se que as regiões mais afetadas pela hantavirose são as Regiões Sul e Centro-Oeste, com ênfase nos Estados do Mato Grosso $(18 \%$ - 219/1.242) e Santa Catarina $(14 \%-179 / 1.242)$ (Figuras 1 B, C). Nessas regiões há a presença de biomas, como o Cerrado, Mata Atlântica e Florestas de Araucárias, ambientes onde habitam os roedores silvestres - reservatórios naturais do hantavírus -, coincidindo as localidades com a maior vulnerabilidade para ocorrência da doença (OLIVEIRA et al., 2015).

Em algumas Regiões observa-se padrões de sazonalidade da doença (Figura 6). Na Região Sul a maior frequência de casos é observada entre os meses de outubro e novembro; já na Região Sudeste verifica-se maior frequência nos meses de abril e maio. Tais padrões sazonais possivelmente estão relacionados à biologia e ao comportamento das diferentes espécies de roedores reservatórios envolvidos na transmissão nas distintas Regiões e biomas brasileiros (DONALISIO et al., 2008; MS, 2013).

Figura 6 - Distribuição dos casos confirmados de hantavirose segundo o mês do início dos sintomas e Região da Unidade Federada da infecção - Brasil, 2007 a 2017

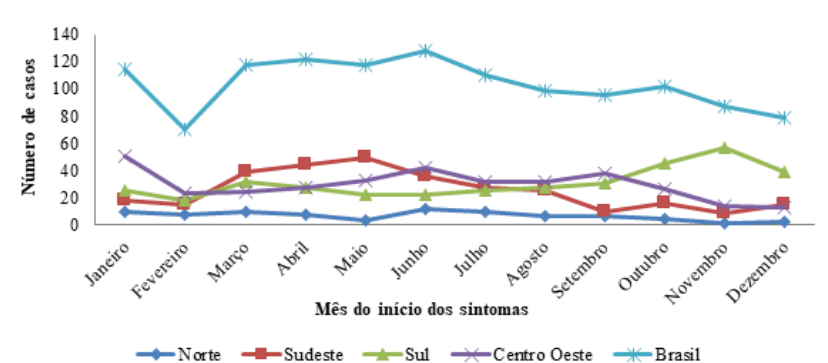

Igualmente, existem diferentes padrões de comportamentos da população humana, que podem expor com maior ou menor frequência as pessoas aos fatores de risco de doenças (OLIVEIRA et al., 2017). Como exemplo destes padrões, observamos casos associados aos períodos de cultivos de grãos na Região Centro-Oeste nos meses mais secos (maio a setembro) (OLIVEIRA et al., 2015). Na região Sul observa-se maior frequência nos meses de outubro a dezembro, período de temperaturas mais elevadas, que favorece situações de risco à infecção, o que tem proporcionado o contato de pessoas aos ambientes silvestres (DE OLIVEIRA, S. V. et al., 2014; OLIVEIRA et al., 2015). Estes episódios sazonais podem estar associados também ao florescimento e à frutificação de bambus ou taquaras, eventos comuns na Região Sul do país. Tal situação é chamada popularmente de "ratada", que é caracterizada pelo aumento da densidade de roedores reservatórios em uma área, propiciado pela oferta de alimentos providos pelas sementes destas taquaras/bambus (OLIVEIRA et al., 2014).

Os prováveis locais de infecção, o perfil agrícola das regiões mais afetadas com seus riscos ambientais (SANTOS et al., 2011; DOS SANTOS et al., 2016, 2017), o crescimento descontrolado das cidades, avançando para áreas de vegetação nativa, e a disponibilidade de alimentos perto de locais de intensa atividade humana, resultaram no deslocamento de reservatórios para áreas próximas às habitações (SANTOS et al., 2011; DOS SANTOS et al., 2016). Os locais prováveis de infecção mais observados neste estudo foram o ambiente de trabalho $(41 \%-511 / 1.242)$ e o domicílio $(29 \%-$ 360/1.242).

Em relação aos óbitos, a maior frequência encontra-se nos moradores de áreas rurais $(71 \%$ $351 / 495)$, corroborando estudo que demostrou que residir em área rural é fator de risco para óbito por hantavirose (WILLEMANN; OLIVEIRA, 2014). Tal fato pode ser explicado pela proximidade com o habitat natural dos hospedeiros (DE OLIVEIRA et al., 2013). Outro aspecto relevante, deve-se ao fato de que o diagnóstico é realizado tardiamente, o que impossibilita uma intervenção médica em tempo hábil, possivelmente por se tratar de uma patologia pouco conhecida pela maioria dos clínicos e pelos profissionais de saúde, o que acarreta na não identificação de muitos casos (DE OLIVEIRA, S. V. et al., 2014). Além disso, muitos óbitos não são registrados como suspeita de hantavirose (OLIVEIRA et al., 2015).

\section{CONSIDERAÇÕES FINAIS}

A hantavirose é uma doença com ampla distribuição geográfica no Brasil e de elevada letalidade, e os casos confirmados e óbitos são detectados mais frequentemente na Região Sul e Centro-Oeste do país ao longo de todos os meses do ano.

Os homens, moradores da zona rural, que exercem atividades de risco, adoecem mais; no entanto a letalidade é maior em mulheres. Assim observa-se, que as atividades laborais que favoreçam o contato de 
pessoas e roedores e os seus ambientes/abrigos, são os fatores de risco para o adoecimento e morte por hantavirose.

O início súbito da doença e o curso clínico inespecífico (assemelhando-se com outras doenças mais frequentes) é um complicador da realização do diagnóstico clínico epidemiológico oportuno. Nesse sentido, faz-se necessária uma padronização do atendimento nos serviços de saúde em relação aos aspectos clínicos e epidemiológicos, com treinamento técnico e educação continuada diante da hantavirose.

Como limitação do estudo, é possível considerar que o banco de dados secundários do Sinan tem muitos registros incompletos ou com baixa qualidade. Desta forma, capacitações das equipes de vigilância epidemiológica deveriam considerar o aprimoramento da informação, que é fundamental para ações de Vigilância em Saúde.

A ação primordial na prevenção da hantavirose é a ampla divulgação da ocorrência da doença, dos seus hospedeiros e seus hábitos, para que o contato com o agente etiológico seja evitado. Os dados apresentados neste estudo destacam a magnitude da hantavirose, expressa no coeficiente de letalidade e amplitude geográfica da sua área de distribuição no Brasil, demonstrando a importância da continuidade, intensificação e ampliação da cobertura do sistema de vigilância epidemiológica da doença no Brasil.

\section{REFERÊNCIAS}

DE OLIVEIRA, S. V. et al. Potential geographic distribution of hantavirus reservoirs in Brazil. PLoS One, 8(12), e85137, 2013.

DE OLIVEIRA, R. C. et al. Hantavirus reservoirs: current status with an emphasis on data from Brazil. Viruses, 6(5), p. 1.929-1.973, 2014.

DE OLIVEIRA, S. V. et al. Análise do perfil epidemiológico da hantavirose no Brasil no período de 2007 a 2012. Rev. Patol. Trop., 43(2), p. 131-142, 2014.

DONALISIO, M. R. et al. Aspectos climáticos em áreas de transmissão de hantavirose no Estado de São Paulo, Brasil. Cad. Saúde Pública. 24, p. 1.141-1.150, 2008.

DOS SANTOS, J. P. et al. Espaço e doença: mudanças antrópicas e a hantavirose. Hygeia. 12(22), p. 62, 2016.

DOS SANTOS, J. P. et al. Does Land Cover Influence the Spatial Distribution of Reservoir Rodent Necromys Lasiurus? SOJ Microbiol. Infect. Dis., 5(4), p. 1-5, 2017.

FERREIRA, M. S. Hantaviruses. Rev. Soc. Bras. Med. Trop., 36(1), p. 81-96, 2003.
FIRTH, C. et al. Diversity and distribution of hantaviruses in South America. J. Virol., 86(24), p. 13.756-13.766, 2012.

ICTV. International Committee on Taxonomy of Viruses. 2016. 023a-cM Hantavirus approved proposal. 2017 March 19. Available from: https://talk.ictvonline.org/ICTV/proposals/2016.023a-cM.A.v2.Hantavirus_sprev.pdf. Cited: 3 June 2019.

KHAN, A. S. et al. Hantavirus pulmonary syndrome. Lancet, 347(9003), p. 739-741, 1996.

MATTAR, S. et al. Diagnosis of hantavirus infection in humans. Expert. Rev. Anti Infect. Ther., 13(8), p. 939-946, 2015.

MS. Ministério da Saúde. Secretaria de Vigilância em Saúde. Departamento de Vigilância Epidemiológica. Manual de vigilância, prevenção e controle das hantaviroses. Brasília: Ministério da Saúde, 2013. 92p.

NICHOL, S. T. et al. Genetic identification of a hantavirus associated with an outbreak of acute respiratory illness. Science, 262(5135), p. 914-917, 1993.

NUNES, M. L. et al. Caracterização clínica e epidemiológica dos casos confirmados de hantavirose com local provável de infecção no bioma Cerrado Brasileiro, 1996 a 2008. Epidemiol. Serv. Saúde, 20(4), p. 537-545, 2011.

OLIVEIRA, S. V. et al. Investigação de ratada associada a florescimento e frutificação de taquaras em São Francisco do Sul, Santa Catarina, Brasil, 2012. Rev. Baiana de Saúde Pública, 37(4), p. 1.071, 2014.

OLIVEIRA, S. V. et al. Vulnerability of Brazilian municipalities to hantavirus infections based on multi-criteria decision analysis. Emerg. Themes Epidemiol., 12(1), p. 15, 2015.

OLIVEIRA, S. V. et al. Climate, Ticks, and Tick-Borne Diseases: Mini Review. Vector Biol. J., 2(1):3, p. 15-17, 2017.

SANTOS, E. D. D.; GARRETT, D. O. Avaliação do sistema de vigilância de hantavírus no Brasil. Epidemiol. Serv. Saúde, 14(1), p. 15-31, 2005.

SANTOS, J. P. D. et al. Land use and occupation and hantavirosis dissemination in the São Sebastião region, Federal District: 2004-2008. Rev. Soc. Bras. Med. Trop., 44(1), p. 53-57, 2011.

SILVA, M. V. D. et al. Hantavirus pulmonary syndrome: report of the first three cases in São Paulo, Brazil. Rev. Soc. Bras. Med. Trop., 39(4), p. 231-234, 1997.

VARGAS, A. et al. Investigação epidemiológica do primeiro caso de hantavirose no Estado do Rio de Janeiro (Brasil Epidemiological investigation of the first case of hantaviruses in the state of Rio de Janeiro, Brazil). J. Health Biol., 4(2), p. 111-116, 2016.

WILLEMANN, M. C. A.; OLIVEIRA, S. V. D. Risk factors associated with hantavirosis fatality: a regional analysis from a case-control study in Brazil. Rev. Soc. Bras. Med. Trop., 47(1), p. 47-51, 2014. 